\title{
Identification of miR-4793-3p as a potential biomarker for bacterial infection in patients with hepatitis $B$ virus-related liver cirrhosis: A pilot study
}

\author{
SHENGLONG LIN ${ }^{1-3}$, MINGHUA LIN ${ }^{1-3}$, HUAXI MA ${ }^{1-3}$, XIANGMEI WANG $^{1-3}$, \\ DONGQING ZHANG ${ }^{1-3}$, WENJUN WU ${ }^{1-3}$, JIAHUANG LIN ${ }^{1-3}$ and HAIBING GAO ${ }^{1-3}$ \\ ${ }^{1}$ Department of Severe Hepatopathy, Mengchao Hepatobiliary Hospital of Fujian Medical University, Fuzhou, Fujian 350002; \\ ${ }^{2}$ Department of Hepatology, Fuzhou Infectious Diseases Hospital, Fuzhou, Fujian 350002; ${ }^{3}$ Department of Hepatology, \\ Infectious Diseases Hospital of Fujian Medical University, Fuzhou, Fujian 350002, P.R. China
}

Received March 22, 2020; Accepted October 9, 2020

DOI: $10.3892 /$ etm.2020.9552

\begin{abstract}
Hepatitis B virus-related liver cirrhosis (HBV-LC) is susceptible to bacterial infections, which could lead to adverse prognosis in patients. MicroRNAs (miRs/miRNAs) are easily detected in peripheral blood and are involved in multiple liver diseases. The present pilot study aimed to investigate differentially expressed (DE) miRNAs in the serum of patients with HBV-LC and bacterial infection, and to identify potential biomarkers. The first batch of clinical samples was collected, including four patients with HBV-LC and infection, four patients with HBV-LC without infection, four patients with chronic hepatitis $\mathrm{B}(\mathrm{CHB})$ and four healthy controls. miRNA expression was analyzed by Affymetrix GeneChip miRNA 4.0 Array. A total of 385 DE miRNAs (upregulated, 160; downregulated, 225) were detected in patients with HBV-LC and infection compared with patients with HBV-LC without infection. miR-4793-3p was significantly upregulated in patients with HBV-LC and infection compared with its levels in the other three groups: HBV-LC without infection [log-transformed fold change $(\operatorname{logFC})=7.96$; $\mathrm{P}=0.0458), \mathrm{CHB}(\log \mathrm{FC}=34.53 ; \mathrm{P}=0.0003)$ and healthy controls ( $\log \mathrm{FC}=3.34 ; \mathrm{P}=0.0219)$ ]. Reverse transcriptionquantitative PCR (RT-qPCR) was performed to validate miR-4793-3p expression in another batch of clinical samples. RT-qPCR showed that miR-4793-3p was highly expressed in patients with HBV-LC and infection compared with its levels in patients with HBV-LC without infection $(\mathrm{P}<0.05)$. The non-
\end{abstract}

Correspondence to: Dr Haibing Gao, Department of Severe Hepatopathy, Mengchao Hepatobiliary Hospital of Fujian Medical University, 312 Xihong Road, Gulou, Fuzhou, Fujian 350002, P.R. China

E-mail: gaohb605@163.com

Key words: bacterial infection, liver cirrhosis, microRNA, microarray, reverse transcription-quantitative, microRNA-4793-3p, TGF- $\beta$ signaling pathway parametric random forest regression model was built to access the diagnostic value of miR-4793-3p, and the receiver operating characteristic curve demonstrated that the area under the curve was $92.2 \%$. Target gene analysis with bioinformatics tools and Gene Expression Omnibus data (GSE46955) showed that miR-4793-3p could participate in the TGF- $\beta$ signaling pathway. Functional experiments revealed that overexpressed miR-4793-3p could impair TGF- $\beta$ function by downregulating Gremlin-1. The present pilot study suggests that miR-4793-3p could be a feasible indicator for bacterial infection in patients with HBV-LC, and it would be valuable for further research.

\section{Introduction}

Worldwide, an estimated 240 million people have chronic hepatitis $B$ virus (HBV) infection, of which the majority of people with chronic HBV infections reside in low- and middleincome countries (1). The Asia-Pacific region has the highest prevalence of HBV infection, with an estimated range from $5-20 \%$ (2). Despite the remarkable progress of HBV vaccination and antiviral treatment, $\mathrm{HBV}$-associated liver diseases remain a significant cause of morbidity and mortality for $\sim 650,000$ deaths annually worldwide (1). Chronic HBV-infected individuals are subjected to an increased risk of adverse sequelae, including liver cirrhosis (LC), decompensated liver disease and hepatocellular carcinoma $(2,3)$. The annual incidence of cirrhosis in chronic HBV-infected patients is $1.0-2.4 \%$, and the annual rate of progression from compensated cirrhosis to decompensated cirrhosis is $\sim 4.6 \%$ (4). The clinical outcome of decompensated cirrhosis is poor, and the 5-year survival rate has decreased from 14 to $35 \%$ (5). Bacterial infection is one of the most relevant complications of cirrhosis, which is the leading cause of hospitalization. Patients with cirrhosis are susceptible to numerous infectious diseases, and the incidence rate of infection is much higher than that among general patients $(6,7)$. It was reported that infection increased mortality by 4 -fold in cirrhosis and contributed to almost $30 \%$ of deaths within 1 month (8). Hence, the study of biomarkers for early prevention, identification and management of infection in patients with cirrhosis is needed. 
MicroRNAs (miRs/miRNAs) are small non-coding RNAs containing $\sim 22$ nucleotides, which can combine with the Argonaute protein family to form the RNA-induced silencing complex (RISC). RISC can bind the 3'- or 5'-untranslated region (UTR) of target mRNAs, leading to RNA silencing and post-transcriptional regulation of gene expression (9). miRNAs are involved in multiple pathophysiological processes and various human diseases. Moreover, as miRNAs can be conveniently isolated from body fluids, they are ideal biomarker candidates. In recent years, an increasing number of studies have provided overwhelming data to support the association of miRNAs with liver diseases, including viral hepatitis, liver cirrhosis, liver failure, steatohepatitis and autoimmune hepatitis (10-13).

Previous studies have confirmed that miRNAs are involved in bacterial infections $(14,15)$. In pulmonary tuberculosis, miR-124 can regulate the toll-like receptor (TLR) signaling pathway by silencing downstream signals such as TLR6, myeloid differentiation primary response 88 (MyD88), TNF receptor-associated factor 6 (TRAF6) and TNF- $\alpha$ (16). Moreover, in mouse macrophage RAW264.7 cells, Salmonella infection could repress let-7a expression and subsequently lead to IL-10 expression, which is a critical anti-inflammatory factor in the immune response (17). Aberrant miRNA expression was also found in liver disease with bacterial infections. Lutz et al (18) revealed that miR-155 is upregulated in the ascites of patients with cirrhosis and spontaneous bacterial peritonitis (SBP), which is associated with adverse short-term survival; and it could be a potential biomarker.

In the present study, the miRNA expression pattern in HBV-related liver cirrhosis (HBV-LC) with bacterial infection was investigated with the aim of understanding the development of the disease and to identify potential miRNA biomarkers for early diagnosis.

\section{Materials and methods}

Sample collection. The present study was ethically approved by Fujian Medical University Mengchao Hepatobiliary Hospital (Fuzhou, China). Written informed consent was obtained from all the participants before sample and data collection. A total of 16 serum samples, including patients with HBV-LC and infection $(n=4)$, patients with HBV-LC without infection $(n=4)$, patients with chronic hepatitis $B$ $(\mathrm{CHB})(\mathrm{n}=4)$ and healthy controls $(n=4)$, were collected to detect differentially expressed (DE) miRNAs. Another 24 serum samples, including eight patients with HBV-LC and infection, eight patients with HBV-LC without infection and eight patients with CHB, were also collected to validate the expression of miRNAs by reverse transcription-quantitative PCR (RT-qPCR). The infection sites of patients with HBV-LC and infection were SBP $(n=6)$, bacterial pneumonia $(n=4)$, coinfection of SBP and pneumonia $(n=3)$ and bacterial urinary infection $(\mathrm{n}=2)$.

The characteristics and clinical information of the participants are summarized in Table I. All patients in the present study had confirmed diagnosis based on the recommendations of the latest guidelines of the Asian Pacific Association for the Study of the Liver for CHB and Liver Fibrosis $(19,20)$. Patients with diagnosis of infection had both clinical symptoms and confirmed bacterial evidence. Positive pathogen culture results were required for the confirming bloodstream, urinary and respiratory infections. For culture-negative pulmonary infections, significant findings of computed tomography scans were needed.

miRNA expression profiling. Total RNA was extracted from $200 \mu \mathrm{l}$ serum using the miRNeasy MicroKit (Qiagen, Inc.) according to the manufacturer's instructions. The purity and concentration of isolated total RNA were tested with a NanoDrop ${ }^{\circledR}$ ND-2000 spectrophotometer (Thermo Fisher Scientific, Inc.) through absorbance measurements at wavelengths of 260 and $280 \mathrm{~nm}$. Only samples with a 260/280 ratio of $\geq 1.6$ were selected. Isolated total RNA samples were stored at $-70^{\circ} \mathrm{C}$ for later use. The Affymetrix GeneChip miRNA 4.0 Array (miRBase version 20.0; Affymetrix; Thermo Fisher Scientific, Inc.) was applied to compare DE miRNAs among HBV-LC with infection, HBV-LC without infection, CHB and healthy controls. Briefly, total RNA $(1 \mu \mathrm{g})$ from each sample was labeled using the FlashTag ${ }^{\mathrm{TM}}$ Biotin HSR RNA labeling kit (according to the Affymetrix user guidelines). The labeled samples were hybridized on miRNA arrays for $18 \mathrm{~h}$ at $48^{\circ} \mathrm{C}$. Arrays were washed to remove non-specifically bound nucleic acids and stained on Fluidics Station 450s (Affymetrix; Thermo Fisher Scientific, Inc.) and then scanned on the GeneChip Scanner 3000 7G system (Affymetrix; Thermo Fisher Scientific, Inc.). Subsequent analysis was conducted through Affymetrix GeneChip Command Console 6.0+ software (Thermo Fisher Scientific, Inc.). Raw data were normalized by the Robust Multi-array Average method to remove systematic variations (21). The raw data were analyzed by Transcriptome Analysis Console software (version 4.0.1; Thermo Fisher Scientific, Inc.), as recommended by Affymetrix. A probe set was considered expressed if $>50 \%$ of samples had a detection above background value below the threshold and $\mathrm{P}<0.05$. The parameter of DE miRNA was defined as a $\log \mathrm{FC}<-1$ or $>1$ with a P-value $<0.05$.

Validation of miRNA array data. Validation of selected DE miRNAs by RT-qPCR was performed as follows: Total miRNA was extracted from patients' serum sample using a Serum/Plasma MicroRNA kit (Aidlab Biotechnologies, Ltd.) according to the manufacturer's instructions. RT of the extracted miRNAs of each sample was conducted with the TaqMan $^{\mathrm{TM}}$ microRNA Reverse Transcription kit (Applied Biosystems; Thermo Fisher Scientific, Inc.). All cDNA samples were kept at $-20^{\circ} \mathrm{C}$ until RT-qPCR analysis. RT-qPCR was performed on a Cobas Z480 machine (Roche Diagnostics) using the TaqMan ${ }^{\mathrm{TM}}$ microRNA Assay kit (Applied Biosystems; Thermo Fisher Scientific, Inc.) according to the manufacturer's protocol. The primers of used are provided in Table SI. The thermocycling conditions were set as follows: Initial activation of Taq polymerase at $95^{\circ} \mathrm{C}$ for $10 \mathrm{~min}$, followed by 40 cycles of PCR amplification at $95^{\circ} \mathrm{C}$ for $15 \mathrm{sec}$ and annealing/elongation at $60^{\circ} \mathrm{C}$ for $1 \mathrm{~min}$. The expression levels of miRNA were normalized to that of U6 small nuclear RNA (snRNA). The relative expression of miRNA was calculated by the $2^{-\Delta \Delta C q}$ formula (22), in which $\Delta \mathrm{Cq}=\mathrm{Cq}$ of miRNA - Cq of U6 snRNA, and $\Delta \Delta \mathrm{Cq}=\Delta \mathrm{Cq}$ of the test group - $\Delta \mathrm{Cq}$ 


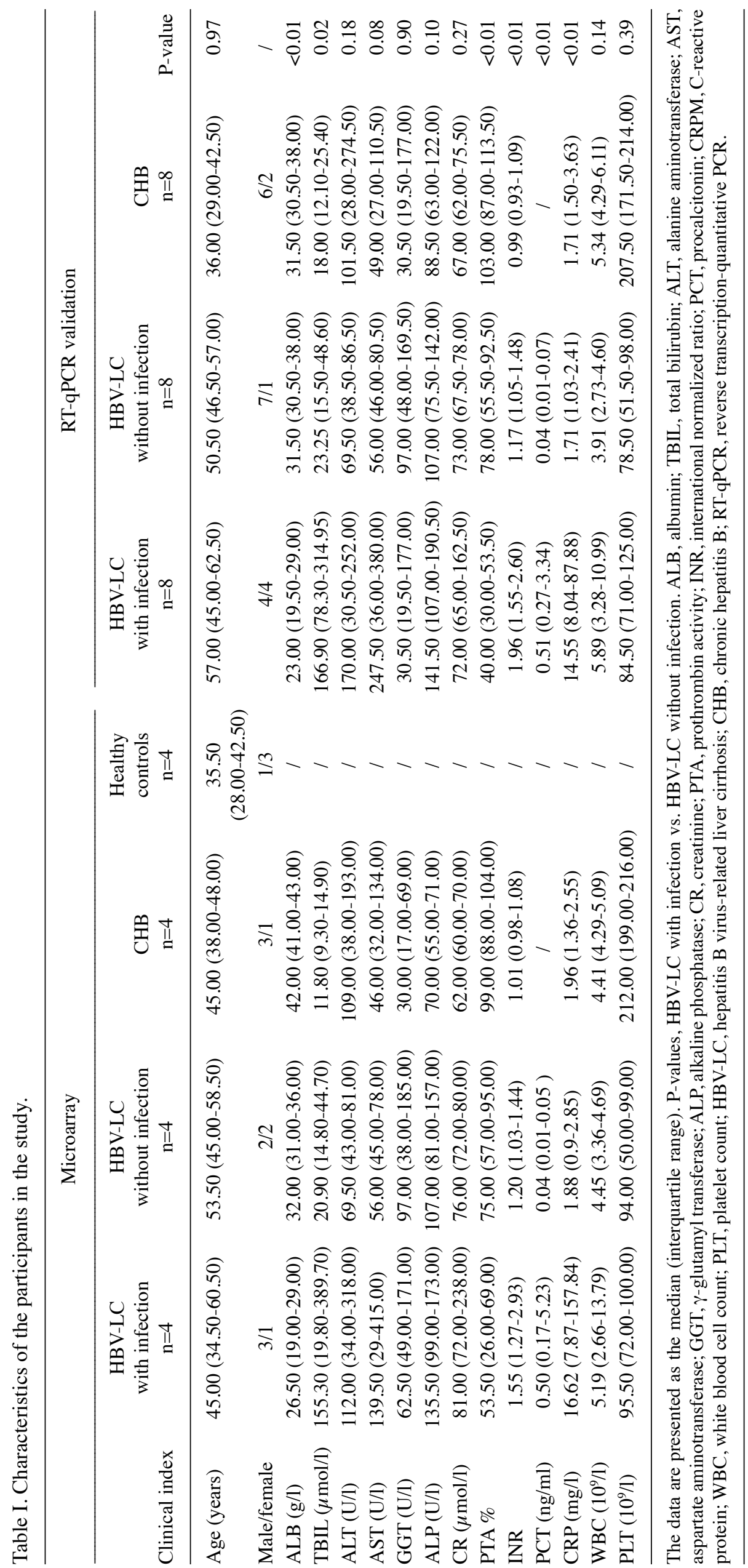


of the control group. The relative quantification values were logarithmically transformed before comparisons.

Dual-Luciferase reporter gene assay. The binding sites between miR-4793-3p and Gremlin-1 (Grem1) 3'-UTR were predicted used the web-based tool TargetScan (http:// www.targetscan.org/). Grem1 3'-UTR or mutant 3'-UTR (3'-UTRmut) fragments were inserted into the psiCHECK-2 plasmid (cat. no. C8011; Promega, Inc.) respectively, using $X b a \mathrm{I}$ and $S a c I$ restriction enzymes. The wild-type or mutant reporter constructs were later co-transfected into 293T cells (cat. no. CRL-11268 ${ }^{\mathrm{TM}}$; American Type Culture Collection). The 293T cells were cultured in RPMI-1640 medium (Gibco; Thermo Fisher Scientific, Inc.) supplemented with 10\% FBS (cat. no. P30-3302; PAN Biotech UK, Ltd.), 1\% penicillinstreptomycin and $0.05 \mathrm{mM} \beta$-mercaptoethanol under a humidified $5 \% \mathrm{CO}_{2}$ atmosphere at $37^{\circ} \mathrm{C}$. miR-4793-3p mimic and specific-miR-4793-3p negative control (NC) were synthesized by General Biol. Prior to transfection, the 293T cells were transferred to 6-well plates until the confluence reached 90-95\%. Grem1 3'-UTR-psiCHECK-2 (1 $\mu \mathrm{g}), 3$ '-UTRmutpsiCHECK-2 $(1 \mu \mathrm{g})$, miR-4793-3p mimic $(50 \mu \mathrm{M})$ or specific-miR-4793-3p NC (50 $\mu \mathrm{M})$ were co-transfected using Lipofectamine ${ }^{\circledR} 2000$ (Invitrogen; Thermo Fisher Scientific, Inc.). The untransfected cells were treated as the normalized control. After transfection, the cells were kept in a $5 \% \mathrm{CO}_{2}$ incubator at $37^{\circ} \mathrm{C}$. After $24 \mathrm{~h}$, the cells were harvested for luciferase activity detection, following the protocol of the dual luciferase reporter assay system (cat. no. E1910; Promega, Inc.). The assay was performed in triplicate.

Cell culture, induction and transfection. THP-1 monocytes (cat. no. TIB-202 ${ }^{\mathrm{TM}}$; American Type Culture Collection) were cultured in RPMI-1640 medium (Gibco; Thermo Fisher Scientific, Inc.) supplemented with $10 \%$ FBS (cat. no. P30-3302; PAN Biotech UK, Ltd.), and $2 \mathrm{mmol} / \mathrm{l}$ L-glutamine under a humidified $5 \% \mathrm{CO}_{2}$ atmosphere at $37^{\circ} \mathrm{C}$. The cells were pre-treated with $20 \mathrm{ng} / \mathrm{ml}$ TGF- $\beta$ (cat. no. HY-P2294; MedChemExpress) for $48 \mathrm{~h}$ and then collected. Firstly, to interrogate the function of TGF- $\beta$ in macrophage differentiation, total cellular RNA was extracted and used to detect macrophage phenotype-related markers by RT-qPCR. Secondly, to investigate the association between miR-4793-3p, Grem1 and the TGF- $\beta$ signaling pathway, TGF- $\beta$-treated THP- 1 cells were transfected with specific miR-4793-3p NC and mimics separately, while non-transfected cells served as the blank control. miRNA-4793-3p mimics $(1.25 \mu \mathrm{l} ; 20 \mu \mathrm{M})$ or miR-4793-3p $\mathrm{NC}(1.25 \mu \mathrm{l} ; 20 \mu \mathrm{M})$ were diluted by $30 \mu \mathrm{l} 1 \mathrm{X}$ ribo $\mathrm{FECT}^{\mathrm{TM}}$ CP Buffer (Guangzhou RiboBio Co., Ltd) and added with $3 \mu \mathrm{l}$ ribo FECT ${ }^{\mathrm{Tм}}$ CP Reagent (Guangzhou RiboBio Co., Ltd) at room temperature for $15 \mathrm{~min}$. The mixture was then added to the prepared TGF- $\beta$-treated THP- 1 cells, kept in a $5 \% \mathrm{CO}_{2}$ incubator at $37^{\circ} \mathrm{C}$. After $36 \mathrm{~h}$, the cells were collected and used to extract total RNA and protein for RT-qPCR and western blotting experiments.

mRNA expression and western blotting. TRIzol ${ }^{\circledR}$ reagent (Invitrogen; Thermo Fisher Scientific, Inc.) was used to obtain total RNA from 293T and THP-1 cells, which was reverse transcribed into cDNA using the SuperScript ${ }^{\mathrm{TM}}$ IV First-
Strand Synthesis System (cat. no. 18091050; Thermo Fisher Scientific, Inc.). Primer information is listed in Table SI. Fluorescent qPCR was performed based on the protocols of the SYBR-GreenER ${ }^{\text {TM }}$ qPCR SuperMix Universal kit (cat. no. 11762500; Thermo Fisher Scientific, Inc.) on the Applied Biosystems 2720 Thermal Cycler (Thermo Fisher Scientific, Inc.). The initial of RT-qPCR was $95^{\circ} \mathrm{C}$ for $10 \mathrm{sec}$, followed by 40 cycles of $95^{\circ} \mathrm{C}$ for $5 \mathrm{sec}$, and then $60^{\circ} \mathrm{C}$ for $30 \mathrm{sec}$. A melting-curve analysis was performed to ensure specificity of the PCR products. The relative gene expression was normalized to $\beta$-actin and calculated by $2-\Delta \Delta \mathrm{Cq}$ formula (22).

The THP-1 cells were lysed to extract cellular protein with $400 \mu 1$ lysis buffer (cat. no. P0013; Beyotime Institute of Biotechnology) for $30 \mathrm{~min}$ on ice. A total of $20 \mu \mathrm{g}$ of cell lysates were separated on $10 \%$ Tris-Glycine eXtended gradient gels (cat. no. 5671093; Bio-Rad Laboratories, Inc.). The targeted proteins were transferred onto $0.2 \mu \mathrm{m}$ nitrocellulose membranes (Cytiva) After transferring proteins, the proteins were blocked with $5 \%$ bovine serum albumin (cat. no. 37525 ; Thermo Fisher Scientific, Inc.) at room temperature for $1 \mathrm{~h}$. Subsequently, the membranes were incubated at $4^{\circ} \mathrm{C}$ overnight with the following primary antibodies: Mouse antibody against Grem1 (1:500; cat. no. sc-515877; Santa Cruz Biotechnology, Inc.) or mouse monoclonal antibody against $\beta$-actin (1:5,000; cat. no. HC201-01; TransGen Biotech Co., Ltd.). Subsequently, the membrane was incubated with ProteinFind ${ }^{\circledR}$ goat anti-mouse $\operatorname{IgG}(\mathrm{H}+\mathrm{L})$, HRP Conjugate (1:10,000; cat. no. HS201-01; TransGen Biotech Co., Ltd.) secondary antibody for $1 \mathrm{~h}$ at room temperature. The protein bands were visualized by enhanced Chemiluminescent detection (SuperSignal ${ }^{\mathrm{TM}}$ West Pico PLUS Chemiluminescent Substrate; cat. no. 34580; Thermo Fisher Scientific, Inc.) and quantified by densitometry using a Gel Imaging System (JS-1070; Shanghai Peiqing Science \& Technology Co., Ltd) for gray value analysis.

Statistical analysis, target gene prediction and functional analysis. The results were analyzed using $\mathrm{R}$ (version 3.6.1; https://cran.r-project.org) and GraphPad Prism (version 8.0 for Windows, GraphPad Software, Inc.). Data are represented as the median and interquartile range. Skewed data were powertransformed to approximate normality. $\mathrm{P}<0.05$ was considered to indicate a statistically significant difference. Levene's test was used for evaluating homogeneity of variance across groups. Student's t-test was used for two-group comparisons, while one-way ANOVA was applied for multiple group comparisons. The Holm correction method was used by the pairwise.t.test function of $\mathrm{R}$ language as the post-hoc test to determine whether the mean difference between specific pairs of group was statistically significant. Since linear regression was not applicable to the small sample size used in the present study, non-parametric random forest regression was applied to build the classification model. A receiver operating characteristic (ROC) curve was plotted to access the diagnostic value of the model. The target genes of miRNAs were predicted with TargetScan and miRDB (http://www.mirdb.org) $(23,24)$. Gene Ontology (GO; Website: http://geneontology.org) and Kyoto Encyclopedia of Genes and Genomes (KEGG; Website: https://www.genome.jp/kegg) enrichment analyses were conducted by the R package, clusterProfiler (version 3.12) (25). Publicly available gene expression data were obtained from 


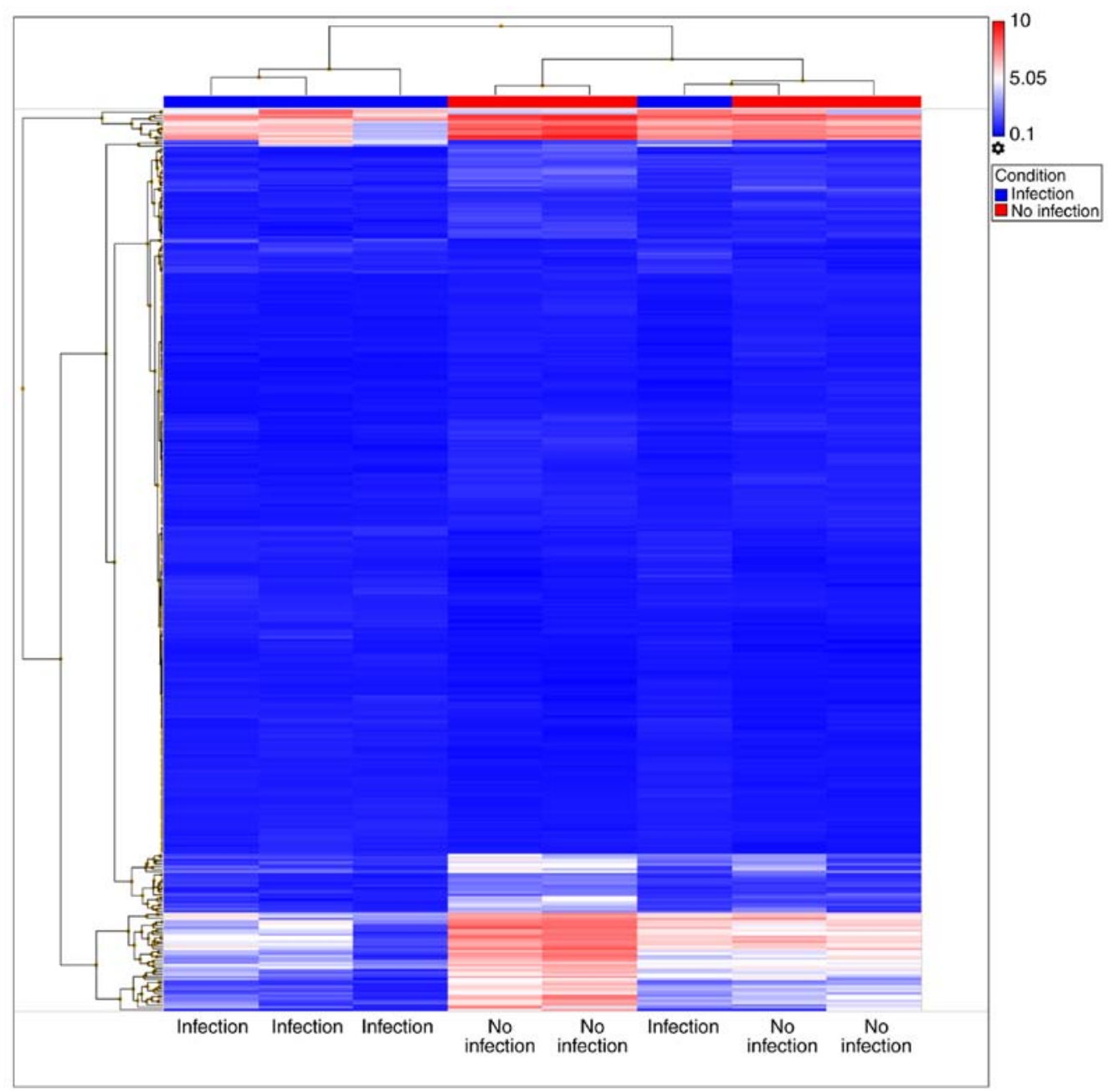

Figure 1. Hierarchical clustering of differential microRNAs. The heatmap shows two-way hierarchical clustering of the differentially expressed microRNAs in the comparison of patients with cirrhosis with and without infection. The rows represent differential microRNAs, and each column represents a sample. The differential microRNA clustering tree is depicted on the left. The blue-to-red color scale illustrates the relative levels of microRNA expression from a higher level to a lower level than that of the reference expression.

the Gene Expression Omnibus (GEO) to analyze the miRNA target genes in bacterial infection. After searching, the dataset GSE46955 was selected, which compared DE genes between patients with bacterial sepsis and healthy controls (26). The relative datasets were downloaded from GEO (https://www. ncbi.nlm.nih.gov/geo/query/acc.cgi?acc=GSE46955).

\section{Results}

Identification of DE miRNA sets. The microarray experiment identified 385 DE miRNAs between the cirrhosis with infection and cirrhosis without infection groups, among which 160 miRNAs were upregulated and 225 were downregulated (Fig. 1). The top five upregulated and the top five downregulated miRNAs are listed in Table II. The available miRNA as a biomarker should express at a high level in patients with cirrhosis and bacterial infection compared to other groups. Under this premise, only miR-4793-3p and miR-4440 matched the criteria. As shown in Table II, miR-4793-3p was upregulated in patients with cirrhosis and infection compared with the levels in patients with cirrhosis without infection $(\log \mathrm{FC}=7.96$; $\mathrm{P}=0.0458)$, patients with $\mathrm{CHB}(\log \mathrm{FC}=34.53 ; \mathrm{P}=0.0003)$ and healthy controls $(\log \mathrm{FC}=3.34 ; \mathrm{P}=0.0219)$. $\mathrm{miR}-4440$ was upregulated in patients with cirrhosis and infection compared with its levels in cirrhosis patients without infection $(\log \mathrm{FC}=7.65 ; \mathrm{P}=0.0408), \mathrm{CHB}(\log \mathrm{FC}=53.11 ; \mathrm{P}=0.0004)$ and healthy controls $(\log \mathrm{FC}=3.18 ; \mathrm{P}=0.0292)$.

Validation and evaluation of miR-4793-3p as a biomarker. RT-qPCR was used to assess the abundance of miR-4793-3p and miR-4440 in another batch of clinical samples. A higher level of miR-4793-3p was detected in patients with cirrhosis and infection compared with those without infection $(\mathrm{P}=0.018)$ and with $\mathrm{CHB}(\mathrm{P}=0.013)$. The expression level of miR-4793-3p in patients with cirrhosis without infection and CHB was not significantly different $(\mathrm{P}=0.397$; data not shown). However, the expression of miR-4440 was not significantly different in validation samples between infection and non-infection groups $(\mathrm{P}<0.05$; data not shown). The expression of miR-4793-3p was consistent with previous microarray data. Non-parametric machine-learning random forest regression was applied to assess the diagnostic value of miR-4793-3p. Previous analysis showed that C-reactive protein (CRP), albumin, international normalized ratio, prothrombin activity, procalcitonin (PCT) 
Table II. Differentially expressed microRNAs in patients with HBV-LC with infection, HBV-LC without infection, CHB and healthy controls.

\begin{tabular}{|c|c|c|c|c|}
\hline Comparisons & ID & Accession & $\log \mathrm{FC}$ & P-value \\
\hline HBV-LC with infection vs. & hsa-miR-4793-3p & MIMAT0019966 & 7.96 & 0.0458 \\
\hline \multirow[t]{9}{*}{ HBV-LC without infection } & hsa-miR-4440 & MIMAT0018958 & 7.65 & 0.0408 \\
\hline & hsa-miR-4668-5p & MIMAT0019745 & 6.97 & 0.0112 \\
\hline & hsa-miR-3613-3p & MIMAT0017991 & 4.69 & 0.0491 \\
\hline & hsa-miR-5093 & MIMAT0021085 & 2.51 & 0.0185 \\
\hline & hsa-miR-6824-5p & MIMAT0027548 & -29.86 & 0.0088 \\
\hline & hsa-miR-6789-5p & MIMAT0027478 & -15.38 & 0.0164 \\
\hline & hsa-miR-4433-3p & MIMAT0018949 & -13.61 & 0.0009 \\
\hline & hsa-miR-6722-3p & MIMAT0025854 & -12.96 & 0.0037 \\
\hline & hsa-miR-6798-5p & MIMAT0027496 & -12.73 & 0.0016 \\
\hline \multirow[t]{10}{*}{ HBV-LC with infection vs. CHB } & hsa-miR-4440 & MIMAT0018958 & 53.11 & 0.0004 \\
\hline & hsa-miR-4793-3p & MIMAT0019966 & 34.53 & 0.0003 \\
\hline & hsa-miR-297 & MIMAT0004450 & 10.37 & 0.0128 \\
\hline & ENSG00000239063 & ENSG00000239063 & 9.44 & 0.0056 \\
\hline & ENSG00000239063 & ENSG00000239063 & 7.28 & 0.0064 \\
\hline & hsa-miR-122-5p & MIMAT0000421 & -23.98 & 0.0297 \\
\hline & hsa-miR-4487 & MIMAT0019021 & -6.29 & 0.0141 \\
\hline & hsa-miR-4270 & MIMAT0016900 & -5.36 & 0.0279 \\
\hline & hsa-miR-1343-5p & MIMAT0027038 & -4.30 & 0.0493 \\
\hline & hsa-miR-4463 & MIMAT0018987 & -3.44 & 0.0293 \\
\hline \multirow[t]{10}{*}{ HBV-LC with infection vs. healthy } & ENSG00000239063 & ENSG00000239063 & 5.06 & 0.0285 \\
\hline & hsa-miR-4532 & MIMAT0019071 & 4.18 & 0.0146 \\
\hline & hsa-miR-4793-3p & MIMAT0019966 & 3.34 & 0.0219 \\
\hline & hsa-miR-4440 & MIMAT0018958 & 3.18 & 0.0292 \\
\hline & hsa-miR-4462 & MIMAT0018986 & 2.75 & 0.0421 \\
\hline & hsa-miR-4487 & MIMAT0019021 & -3.53 & 0.0448 \\
\hline & hsa-miR-1298-3p & MIMAT0026641 & -1.35 & 0.0104 \\
\hline & hsa-miR-3675-3p & MIMAT0018099 & -1.31 & 0.0182 \\
\hline & hsa-mir-3924 & MI0016432 & -1.29 & 0.0102 \\
\hline & ENSG00000252154 & ENSG00000252154 & -1.28 & 0.0008 \\
\hline
\end{tabular}

The top five upregulated and top five downregulated microRNAs in each comparison group are presented in the table. HBV, hepatitis B virus; LC, liver cirrhosis; $\log \mathrm{FC}, \log$-transformed fold change.

and total bilirubin were associated with infections in patients with cirrhosis (Table I). These variables, together with miR-4793-3p, were used to build the random forest model. As demonstrated in the ROC plot, the model had an area under the curve (AUC) of $92.2 \%$. The plot of variable importance showed that CRP was the most critical factor (Fig. 2).

Target genes and functional prediction. A total of 383 potential target genes of miR-4793-3p were both found in TargetScan $(n=4,995)$ and miRDB $(n=424)$. GO and KEGG enrichment analyses were conducted on these 383 genes. The GO analysis demonstrated that the target genes clustered in cellular components of 'nuclear speck', 'nuclear lamina' and 'ribbon synapse'; in the molecular functions of 'modification-dependent protein binding', 'nuclear export signal receptor activity', 'receptor- regulated SMAD binding' and 'nucleocytoplasmic carrier activity' (Fig. 3A and B). The KEGG analysis showed that the target genes were also involved in many signaling pathways (Fig. 3C), and most enriched in the pathways of "prolactin signaling pathway', 'TGF- $\beta$ signaling pathway', 'pancreatic cancer' and 'hepatitis B' (Fig. 3D). Meanwhile, a previously reported gene expression data of patients with bacterial sepsis was downloaded from the GEO database (GSE46955). After analyzing the data, 432 downregulated DE genes associated with bacterial infection were obtained (Table SII). Among them, five genes are also found in the 383 potential target genes list, including Grem1, progestin and adipoQ receptor family member 4 (paqr4), calnexin (canx), macrophage scavenger receptor 1 (msrl) and zinc binding alcohol dehydrogenase domain containing 2 (zadh2) (Fig. 4 and Table III). 

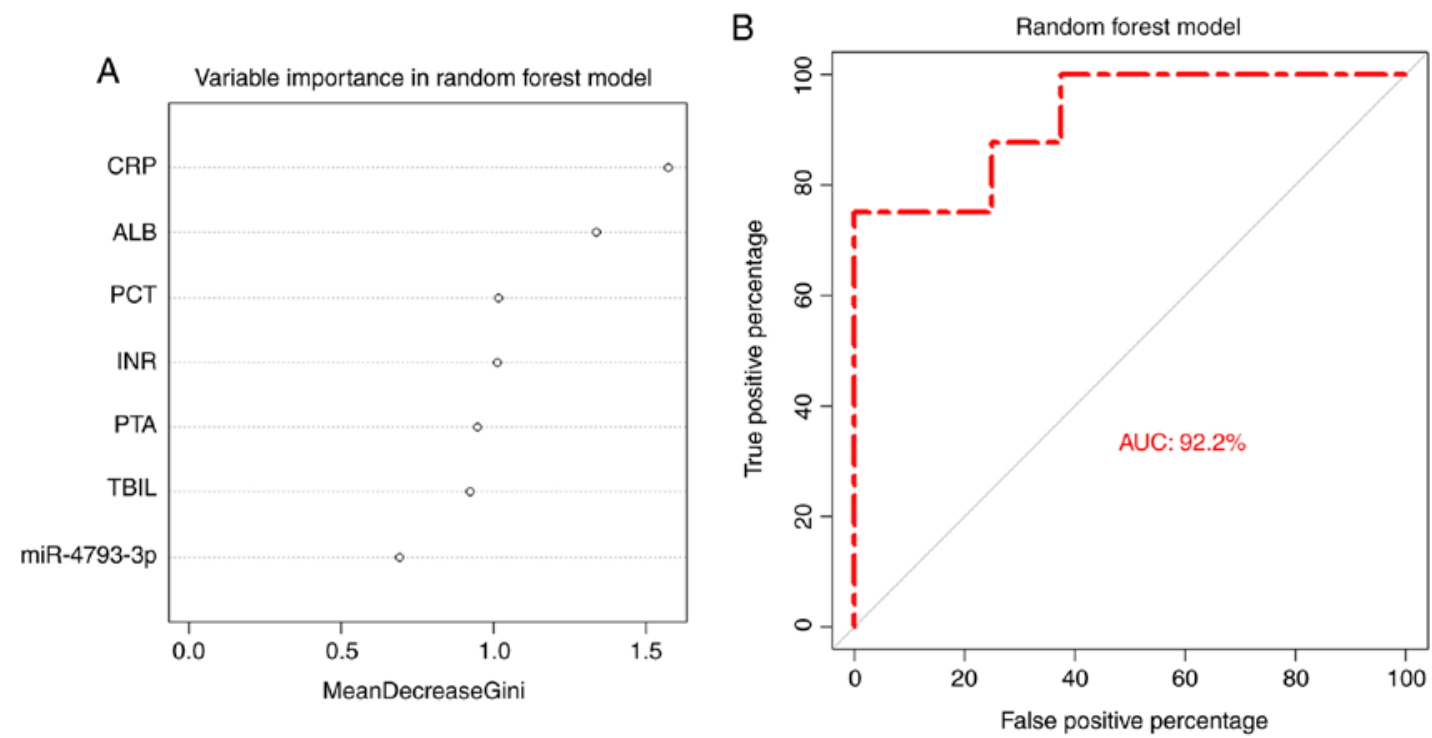

Figure 2. Variable importance plot and ROC curve of the random forest model. (A) The plot of variable importance presents that C-reactive protein is the most critical factor in the model. (B) The AUC of the ROC plot is 92.2\%. CRP, C-reactive protein; ALB, albumin; INR, international normalized ratio; PTA prothrombin activity; PCT, procalcitonin; TBIL, total bilirubin; ROC, receiver operating characteristic; AUC, area under the curve; miR, microRNA.

Table III. Expression of Grem1, paqr4, canx, msr1 and zadh2 in the public dataset GSE46955.

\begin{tabular}{lcccccc}
\hline Gene & logFC & AveExpr & $\mathrm{t}$ & P-value & adj.P.Val & B \\
\hline msr1 & -1.90 & 7.04 & -12.59 & $1.67 \times 10^{-9}$ & $4.62 \times 10^{-7}$ & 12.21 \\
paqr4 & -1.47 & 8.23 & -6.92 & $4.32 \times 10^{-6}$ & $8.60 \times 10^{-5}$ & 4.31 \\
canx & -1.27 & 10.47 & -6.56 & $8.02 \times 10^{-6}$ & $1.32 \times 10^{-4}$ & 3.68 \\
zadh2 & -1.32 & 8.03 & -4.95 & $1.62 \times 10^{-4}$ & $1.22 \times 10^{-3}$ & 0.63 \\
Grem1 & -2.55 & 7.49 & -4.22 & $7.03 \times 10^{-4}$ & $3.59 \times 10^{-3}$ & \\
-0.86 & & & & & \\
\hline
\end{tabular}

Grem1, Gremlin 1; paqr4, progestin and adipoQ receptor family member 4; canx, calnexin; msr1, macrophage scavenger receptor 1; zadh2, zinc binding alcohol dehydrogenase domain containing 2; logFC log-transformed fold change; AveExpr, average expression; adj.P.Value, adjusted P-value; $\mathrm{B}$, the log-odds of differential expression.

Overexpressed miR-4793-3p can impair the TGF- $\beta$ pathway by targeting Grem1. In response to pathogens, macrophages can dichotomize into M1-like macrophage as a pro-inflammatory phenotype or M2-like macrophage as an anti-inflammatory phenotype (27). As reported in previous studies, TGF- $\beta$ could induce macrophages into the M2 phenotype (28-30), which was consistent with the present study. Compared with the control group, M2-related makers such as C-X-C motif chemokine receptor 4; IL-10 and arginase 1 were upregulated, while M1-related makers such as human leukocyte antigen-DR isotype, IL-12, CD80, inducible nitric oxide synthase, TNF- $\alpha$ and monocyte chemoattractant protein were significantly downregulated in TGF- $\beta$-treated THP-1 cells (Fig. 5A). Smad 2 and Smad 3 are known as the downstream genes of the TGF- $\beta$ pathway. Following TGF- $\beta$ stimulus, together with Smad2 and Smad3, Grem1 was also expressed in relatively compared with the control group, which indicated that Grem1 could be activated by TGF- $\beta$. However, when miR-4793-3p mimics were transfected into TGF- $\beta$-treated THP- 1 cells, the expression of Smad2,
Smad3 and Grem1 was significantly reduced (Fig. 5B). This suggested that overexpressed miR-4793-3p could negatively regulate the TGF- $\beta$ pathway by targeting Grem 1 .

Greml is validated as the target of miR-4793-3p. To confirm the association between miR-4793-3p and Grem1, a dual luciferase reporter assay was performed. As shown in Fig. 6A, the relative luciferase activity was significantly declined when 293T cells were co-transfected with Grem1-wild-type and miR-4793-3p mimics compared with the NC group. However, no significant difference was observed when cells were co-transfected with Grem1-mutant and miR-4793-3p mimics compared with the levels in the NC group. Significantly higher expression of miR-4793 was detected in 293T cells transfected with miR-4793-3p mimics compared with cells transfected with miR-4793-3p mimic NC or the control (Fig. 6B). Overexpressed miR-4793-3p could downregulate Grem1 expression both at the mRNA and protein levels (Fig. 6C and D). These results provided evidence that miR-4793-3p could bind to the $3^{\prime}-$ UTR of Grem1 and negatively regulate Grem1 expression. 

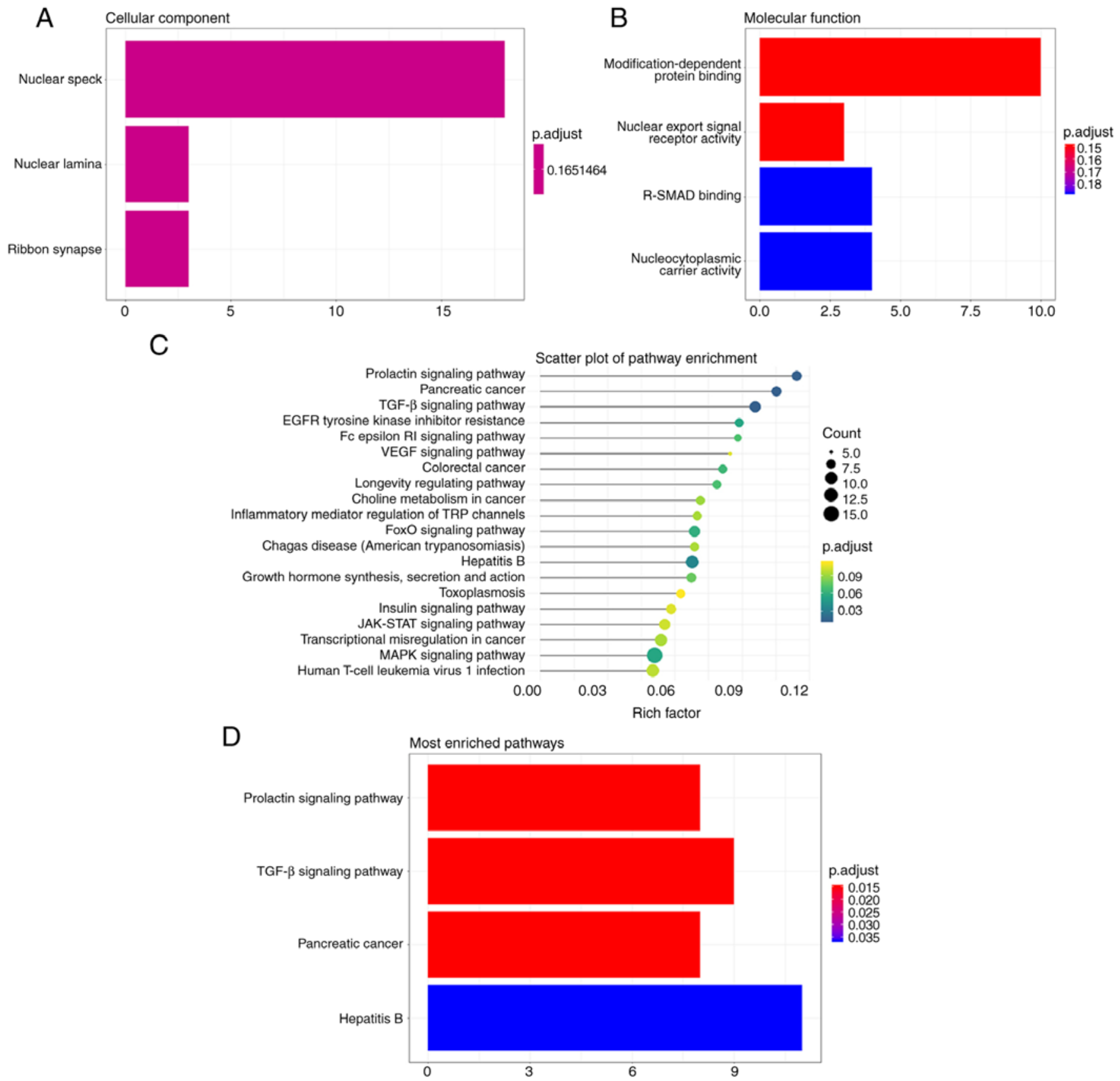

Figure 3. GO and KEGG enrichment analyses for predicted target genes of miR-4793-3p. The plots representing the (A) cellular component and (B) molecular function of GO analysis. The y-axis represents enriched GO terms, and the $\mathrm{x}$-axis represents the number of clustered genes. (C) Scatter plot showing the result of KEGG analysis. (D) Bar plot showing the most enriched pathway terms. The y-axes of the plots in panels C and D represent the KEGG pathway terms. The bubble size in panel $\mathrm{C}$ and the $\mathrm{x}$-axis of the plot in panel D represent the number of clustered genes. GO, Gene Ontology; KEGG, Kyoto Encyclopedia of Genes and Genomes; R-SMAD; receptor-regulated SMAD; TRP, transient receptor potential.

\section{Discussion}

Early detection of bacterial infection is crucial for patients with cirrhosis. However, the broad use of biomarkers in cirrhosis such as CRP and procalcitonin for bacterial infections has several limitations, and the study results remain controversial (7). A recently reported biomarker, soluble CD14 subtype (presepsin), shows a favorable outcome in patients with cirrhosis and bacterial infection, which requires more supporting data (31). The present study investigated DE miRNAs in patients with HBV-related cirrhosis and bacterial infections. As presented, miR-4793-3p was upregulated in patients with cirrhosis and infection in both the microarray experiment and RT-qPCR validation. Thus, miR-4793 could be a feasible biomarker for patients with cirrhosis and bacterial infections.

In the GO and KEGG enrichment analyses, the predicted target genes of miR-4793-3p were mostly enriched in the TGF- $\beta$ signaling pathway. TGF- $\beta$ is critical in the innate immune system. While TGF- $\beta$ serves as an anti-inflammatory factor, which could suppress the augmentation of bacterial infection, overexpressed TGF- $\beta$ could also impair the immune clearance of the pathogen (32). A previous study demonstrated that TGF- $\beta$ could induce macrophages to transform into the 


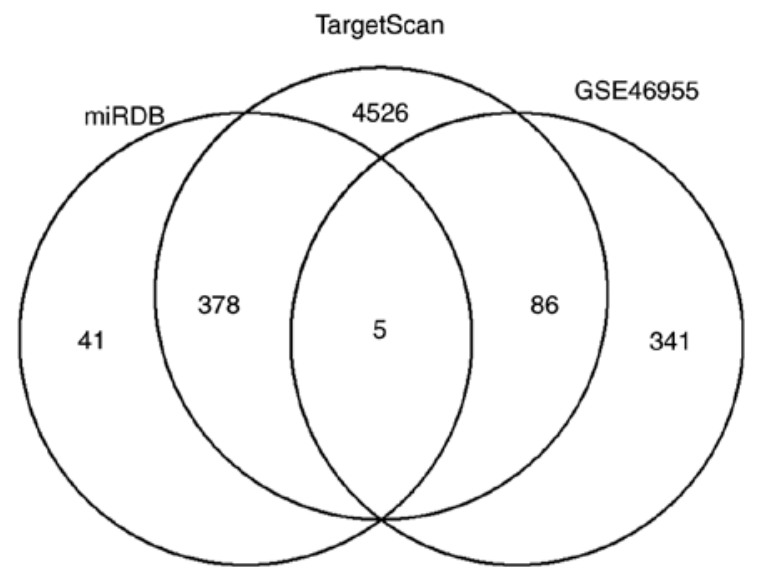

Figure 4. Venn diagram of TargetScan, miRDB and GSE46955 analysis. The predicted target genes of miR-4793-3p in TargetScan $(n=4,995)$ and miRDB $(\mathrm{n}=424)$ were compared with the downregulated differentially expressed genes in the Gene Expression Omnibus dataset GSE46955 ( $n=432$ ). The intersection of three gene lists consists of five genes, including Gremlin-1, adipoQ receptor family member 4 , calnexin, macrophage scavenger receptor 1 and zinc binding alcohol dehydrogenase domain containing 2 .

M2 phenotype, which is anti-inflammatory rather than proinflammatory (28). Upregulated TGF- $\beta$ was associated with the lack of maturation of NK cells in infant mice, which are prone to viral infection in early life (33). Endogenous TGF- $\beta$ modulates NK cell responses to cytokines and TLR agonists. Downregulated endogenous TGF- $\beta$ expression significantly induced NK cells to produce interferon-gamma (IFN- $\gamma$ ), amplifying the innate immune response to pathogens (34). Moreover, TGF- $\beta$ could also regulate Th17 and Treg cell differentiation by inducing $\mathrm{T}$ cell-related factor forkhead box protein P3 expression (35). TGF- $\beta$ was reported to participate in bacterial infection. Overexpressed TGF- $\beta$ in a sepsis mouse model could protect the host from bacterial infection (36). TLR4, known as a critical receptor for pathogen-associated molecular patterns, can be stimulated by lipopolysaccharide from Gram-negative bacteria and initiate the innate immune defense against bacterial attack (37). Smad6, one of the inhibitory SMADs of TGF- $\beta$ signaling, could inhibit the TLR4 signaling pathway by disrupting the Pellino-1-mediated TLR4 signaling complex, leading to anti-inflammatory activity (38). Intriguingly, it was also reported that miR-4793-3p expressed inversely to TLR4 gene expression in necrotizing enterocolitis, suggesting that miR-4793-3p may be associated with the TLR4 pathway (39). The present study proposed that miR-4793-3p could play a role in the innate immune response as part of the TGF- $\beta$ signaling pathway.

To further evaluate the function of miR-4793-3p, public gene expression data of patients with bacterial-induced sepsis were obtained from the GEO database (GSE46955). After analyzing the data, a total of 432 downregulated genes associated with bacterial infection were obtained. Comparing the potential target genes of miR-4793-3p and the public gene expression data, the present study found five genes that were common in two gene lists, including Grem1, paqr4, canx, msr1 and zadh2. Grem1 encodes Gremlin-1 protein, which is a member of the bone morphogenic protein antagonist family. TGF- $\beta$ stimulates Grem1 expression in bronchial and kidney
A
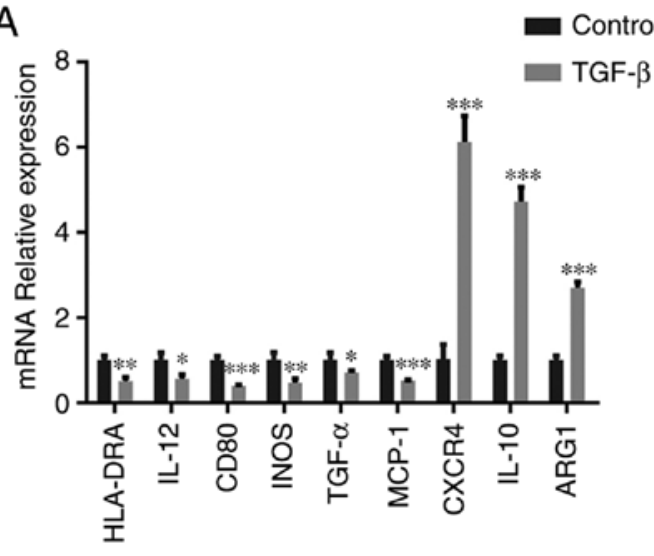

B

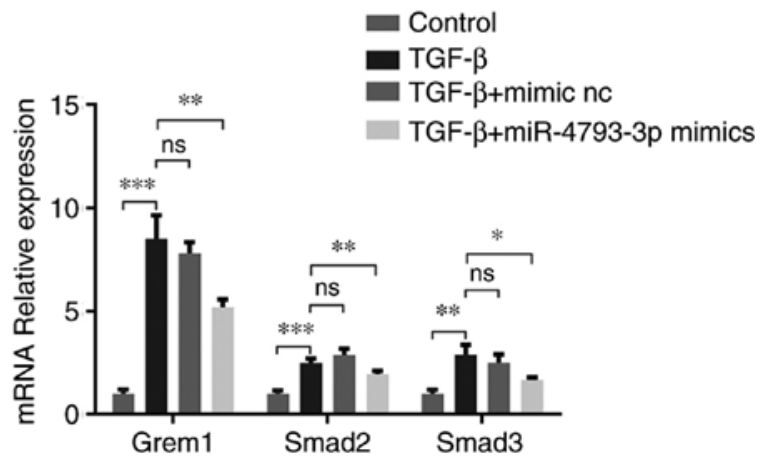

Figure 5. miR-4793-3p negatively regulates the TGF- $\beta$ pathway. (A) In TGF- $\beta$-treated THP-1 cells, the expression levels of M2-related makers, CXCR4, IL-10 and ARG1, were significantly upregulated. By contrast, the expression levels of M2-related makers, HLA-DR, IL-12, CD80, iNOS, TNF- $\alpha$ and MCP-1, were significantly downregulated. (B) TGF- $\beta$-treated THP-1 cells increased Grem1, Smad2 and Smad3 levels compared with controls. When the cells were transfected with miR-4793-3p mimics, the expression levels of Grem1, Smad2 and Smad3 significantly decreased. There was no significant change in the expression levels of Grem1, Smad2 or Smad3 in the specific miR-4793-3p mimic NC group compared with controls. ${ }^{*} \mathrm{P}<0.05 ;{ }^{* *} \mathrm{P}<0.01$ and ${ }^{* * *} \mathrm{P}<0.001$. CXCR4, C-X-C motif chemokine receptor 4; ARG1, arginase 1; HLA-DR, human leukocyte antigen-DR isotype; iNOS, inducible nitric oxide synthase; MCP1, monocyte chemoattractant protein; NC, negative control; ns, not significant; miR, microRNA.

epithelial cells, which could promote tissue fibrosis (40). In a study of cervical cancer, miR-137 could suppress the TGF- $\beta$ signaling pathway by downregulating Grem1 (41). These findings are consistent with the present study, as the expression of Grem 1, Smad 2 and Smad 3 were upregulated in TGF- $\beta$-treated THP-1 cells, suggesting that Grem 1 could be a downstream gene of the TGF- $\beta$ signaling pathway. In the present study, Grem1 was validated as a target gene of miR-4793-3p. Overexpressed miR-4793-3p could reduce Grem1 expression at the mRNA and protein levels. Therefore, upregulated miR-4793-3p in patients with cirrhosis and bacterial infection could suppress the TGF- $\beta$ signaling pathway via binding to Grem1, which could augment bacteria-induced inflammation and aggravate infection.

Certain limitations existed in the present study. In the microarray analysis, the DE miRNA results could be affected by the small sample size. The hierarchical clustering of the microarray data showed that one patient with LC and bacterial infection was assigned to the non-infection group. It could be that the inter-patient variation was quite high, making the 
A

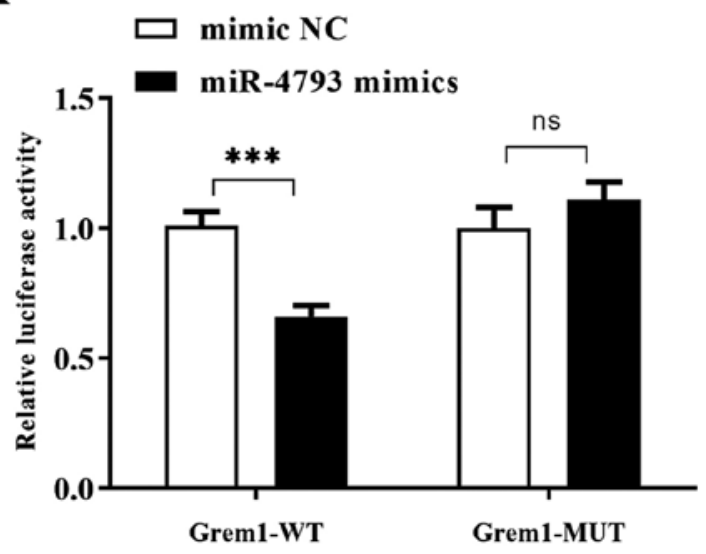

C

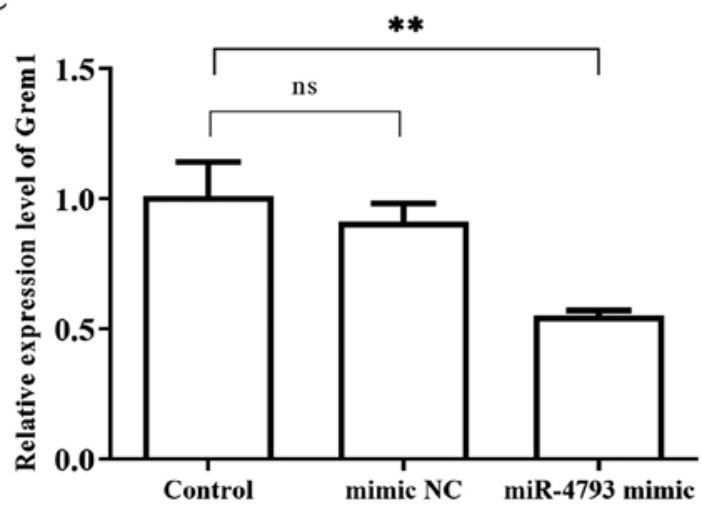

B

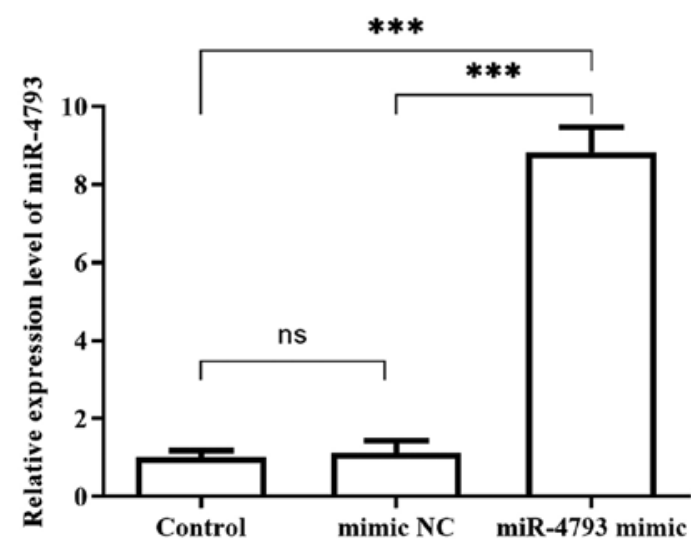

D

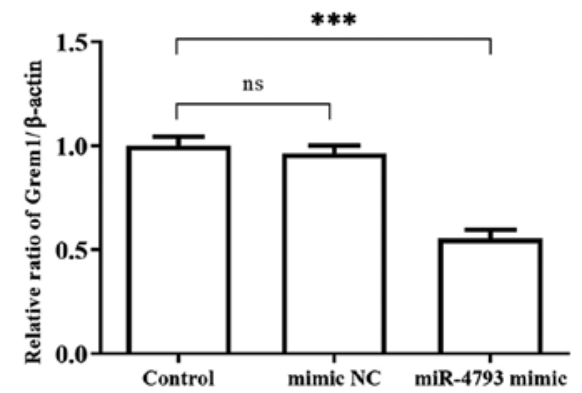

Grem1

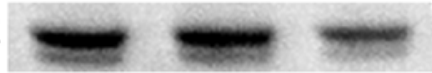

$\beta$-actin

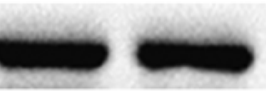

Control

Figure 6. Grem1 is a target gene of miR-4793-3p. (A). Dual-Luciferase assay demonstrated significantly declined luciferase activity in the 293T cells, which were co-transfected with Grem1-WT and miR-4793-3p mimics compared with mimic-NC. By contrast, no significant change in luciferase activity was observed in cells co-transfected with Grem1-MUT and miR-4793-3p mimics compared with mimic-NC. (B) Significantly higher expression of miR-4793 was detected by fluorescent reverse transcription-quantitative PCR in 293T cells transfected with miR-4793-3p mimics compared with cells transfected with miR-4793-3p mimic NC or the control. No significant difference was observed between miR-4793-3p mimic NC and the control groups. (C) In TGF- $\beta$-treated THP-1 cells transfected with miR-4793-3p mimics, significantly downregulated Grem1 mRNA levels was observed compared with the control. By contrast, the mRNA levels of Grem1 was not significantly different between the miR-4793-3p mimic NC group and the control. (D) Western blotting showed significantly downregulated Grem 1 protein levels in the miR-4793-3p mimics group compared with the control. There was no significant change in Grem1 protein levels in the miR-4793-3p mimic NC group compared the control. $\beta$-actin served as the internal reference gene. ${ }^{* *} \mathrm{P}<0.01$ and ${ }^{* * *} \mathrm{P}<0.001$. WT, wild-type; MUT, mutant; NC, negative control; ns, not significant; miR, microRNA; Grem1, Gremlin-1.

distinction difficult. However, despite inter-patient variability, the consistent differential expression effects were detected in the inferred group. Moreover, not all DE miRNAs were validated by RT-qPCR in clinical patients, due to a shortage of research funding. The diagnostic value of miR-4793-3p was analyzed with non-parametric random forest regression, which also requires large samples to build a reliable model. Lastly, not all the potential targeted genes of miR-4793-3p were analyzed in the present study. Notably, macrophage scavenger receptor 1 (msrl), also known as class A scavenger receptor (SR-A), is a multifunctional receptor which is expressed primarily on macrophages (42). It was reported to be associated with macrophage polarization, the innate immune response to autoimmune conditions and pathogens-associated molecular patterns (43). Mrs1-deficient mice were susceptible to pneumococcal pneumonia $(44,45)$. Meanwhile, the mRNA levels of mrs1 and mannose receptor $\mathrm{C}$ type 1 were reduced in influenza virus infection, which could lead to impairment of macrophage phagocytosis and secondary bacterial infection (46). Thus, the association between miR-4793-3p and mrs1 in HBV-related cirrhosis with bacterial infection requires further investigation.

In the present pilot study, microarray was performed to detect highly expressed miR-4793-3p in patients with cirrhosis and bacterial infection. Combined with bioinformatics and public data analysis, miR-4793-3p was found to participate in the TGF- $\beta$ signaling pathway. Functional experiments demonstrated that miR-4793-3p could negatively regulate the TGF- $\beta$ pathway via binding to Grem 1 . Therefore, miR-4793-3p could be a potential biomarker for bacterial infection in HBV-related cirrhosis, and it would be worthy of further investigation. 


\section{Acknowledgements}

Not applicable.

\section{Funding}

The present study was supported by the grants of Middle-aged and Young Talents in Health Science Fostering Project of Fujian Province, PRC (grant no. 2016-ZQN-77); the Natural Science Foundation of Fujian Province, PRC (grant no. 2017J01160); the Key Clinical Specialty Discipline Construction Program of Fuzhou, Fujian Province, PRC (grant no. 201510301) and the Infectious Disease Medical Center Construction Program of Fuzhou, Fujian Province, P.R. China (grant no. 2018080306).

\section{Availability of data and materials}

The datasets generated and analyzed during the current study are available in the GEO repository (https://www.ncbi.nlm. nih.gov/geo/query/acc.cgi?acc=GSE156347).

\section{Authors' contributions}

SL and HG designed the study, analyzed the data and wrote the manuscript. HM, WW and JL recruited the participates and collected the samples. XW and DZ performed the experiments. ML analyzed and interpreted the data, revised and edited the manuscript. All authors read and approved the final manuscript.

\section{Ethics approval and consent to participate}

The present study complied with the Declaration of Helsinki and was ethically approved by Fujian Medical University Mengchao Hepatobiliary Hospital (Fuzhou, China). Written informed consent was obtained from all the participants before sample and data collection.

\section{Patient consent for publication}

Not applicable.

\section{Competing interests}

The authors declare that they have no competing interests.

\section{References}

1. Guidelines WHO; Approved by the Guidelines Review Committee: In: Guidelines for the Prevention, Care and Treatment of Persons with Chronic Hepatitis B Infection. World Health Organization Copyright (C) World Health Organization 2015., Geneva2015.

2. Lesmana LA, Leung NWY, Mahachai V, Phiet PH, Suh DJ, Yao $\mathrm{G}$ and Zhuang H: Hepatitis B: Overview of the burden of disease in the Asia-Pacific region. Liver Int 26 (S2): 3-10, 2006.

3. Lin X, Robinson NJ, Thursz M, Rosenberg DM, Weild A, Pimenta JM and Hall AJ: Chronic hepatitis B virus infection in the Asia-Pacific region and Africa: Review of disease progression. J Gastroenterol Hepatol 20: 833-843, 2005.

4. Guan R and Lui HF: Treatment of hepatitis B in decompensated liver cirrhosis. Int J Hepatol 2011: 918017, 2011. https://doi. org/10.4061/2011/918017.
5. Peng CY, Chien RN and Liaw YF: Hepatitis B virus-related decompensated liver cirrhosis: Benefits of antiviral therapy. J Hepatol 57: 442-450, 2012.

6. Bajaj JS, O'Leary JG, Wong F, Reddy KR and Kamath PS: Bacterial infections in end-stage liver disease: Current challenges and future directions. Gut 61: 1219-1225, 2012.

7. Jalan R, Fernandez J, Wiest R, Schnabl B, Moreau R, Angeli P, Stadlbauer V, Gustot T, Bernardi M, Canton R, et al: Bacterial infections in cirrhosis: A position statement based on the EASL Special Conference 2013. J Hepatol 60: 1310-1324, 2014.

8. Arvaniti V, D'Amico G, Fede G, Manousou P, Tsochatzis E, Pleguezuelo $M$ and Burroughs AK: Infections in patients with cirrhosis increase mortality four-fold and should be used in determining prognosis. Gastroenterology 139: 1246-1256. e1245, 2010.

9. Dueck A, Ziegler C, Eichner A, Berezikov E and Meister G: microRNAs associated with the different human Argonaute proteins. Nucleic Acids Res 40: 9850-9862, 2012.

10. Loosen SH, Schueller F, Trautwein C, Roy S and Roderburg C: Role of circulating microRNAs in liver diseases. World J Hepatol 9: 586-594, 2017.

11. Starkey Lewis PJ, Dear J, Platt V, Simpson KJ, Craig DG, Antoine DJ, French NS, Dhaun N, Webb DJ, Costello EM, et al: Circulating microRNAs as potential markers of human druginduced liver injury. Hepatology 54: 1767-1776, 2011.

12. DiStefano JK and Gerhard GS: Circulating microRNAs in nonalcoholic fatty liver disease. Expert Rev Gastroenterol Hepatol 10: 161-163, 2016.

13. Migita K, Komori A, Kozuru H, Jiuchi Y, Nakamura M, Yasunami M, Furukawa H, Abiru S, Yamasaki K, Nagaoka S, et al: Circulating microRNA profiles in patients with type-1 autoimmune hepatitis. PLoS One 10: e0136908, 2015.

14. Das K, Garnica O and Dhandayuthapani S: Modulation of Host miRNAs by intracellular bacterial pathogens. Front Cell Infect Microbiol 6: 79, 2016.

15. Zhou $\mathrm{X}, \mathrm{Li} \mathrm{X}$ and $\mathrm{Wu} \mathrm{M}$ : miRNAs reshape immunity and inflammatory responses in bacterial infection. Signal Transduct Target Ther 3: 14, 2018.

16. Ma C, Li Y, Li M, Deng G, Wu X, Zeng J, Hao X, Wang X, Liu J, Cho WC, et al: microRNA-124 negatively regulates TLR signaling in alveolar macrophages in response to mycobacterial infection. Mol Immunol 62: 150-158, 2014.

17. Schulte LN, Eulalio A, Mollenkopf HJ, Reinhardt R and Vogel J: Analysis of the host microRNA response to Salmonella uncovers the control of major cytokines by the let-7 family. EMBO J 30: 1977-1989, 2011.

18. Lutz P, M Haimid M, Pohlmann A, Lehmann J, Jansen C, Schierwagen R, Klein S, Strassburg CP, Spengler U and Trebicka J: MicroRNA-155 is upregulated in ascites in patients with spontaneous bacterial peritonitis. Sci Rep 7: 40556, 2017.

19. Sarin SK, Kumar M, Lau GK, Abbas Z, Chan HL, Chen CJ, Chen DS, Chen HL, Chen PJ, Chien RN, et al: Asian-Pacific clinical practice guidelines on the management of hepatitis B: A 2015 update. Hepatol Int 10: 1-98, 2016.

20. Shiha G, Sarin SK, Ibrahim AE, Omata M, Kumar A, Lesmana LA, Leung N, Tozun N, Hamid S, Jafri W, et al; Jury of the APASL Consensus Development Meeting 29 January 2008 on Liver Fibrosis With Without Hepatitis B or C: Liver fibrosis: Consensus recommendations of the Asian Pacific Association for the Study of the Liver (APASL). Hepatol Int 3: 323-333, 2009.

21. Bolstad BM, Irizarry RA, Astrand M and Speed TP: A comparison of normalization methods for high density oligonucleotide array data based on variance and bias. Bioinformatics 19: 185-193, 2003.

22. Livak KJ and Schmittgen TD: Analysis of relative gene expression data using real-time quantitative PCR and the 2(-Delta Delta C(T)) Method. Methods 25: 402-408, 2001.

23. Agarwal V, Bell GW, Nam JW and Bartel DP: Predicting effective microRNA target sites in mammalian mRNAs. eLife 42015.

24. Liu W and Wang X: Prediction of functional microRNA targets by integrative modeling of microRNA binding and target expression data. 20: 18, 2019.

25. Yu G, Wang LG, Han Y and He QY: clusterProfiler: An R package for comparing biological themes among gene clusters. OMICS 16: 284-287, 2012. 
26. Shalova IN, Lim JY, Chittezhath M, Zinkernagel AS, Beasley F, Hernández-Jiménez E, Toledano V, Cubillos-Zapata C, Rapisarda A, Chen J, et al: Human monocytes undergo functional re-programming during sepsis mediated by hypoxia-inducible factor-1 $\alpha$. Immunity 42: 484-498, 2015.

27. Atri C, Guerfali FZ and Laouini D: Role of human macrophage polarization in inflammation during infectious diseases. Int $\mathrm{J}$ Mol Sci 19: 1801, 2018

28. Zhang F, Wang H, Wang X, Jiang G, Liu H, Zhang G, Wang H, Fang R, Bu X, Cai S, et al: TGF- $\beta$ induces M2-like macrophage polarization via SNAIL-mediated suppression of a pro-inflammatory phenotype. Oncotarget 7: 52294-52306, 2016.

29. Song X, Xie S, Lu K and Wang C: Mesenchymal stem cells alleviate experimental asthma by inducing polarization of alveolar macrophages. Inflammation 38: 485-492, 2015.

30. Saha B, Kodys K, Szabo G and Hepatitis C: Hepatitis C virus-induced monocyte differentiation into polarized M2 macrophages promotes stellate cell activation via TGF- $\beta$. Cell Mol Gastroenterol Hepatol 2: 302-316.e8, 2016.

31. ischer P, Grigoras C, Bugariu A, Nicoara-Farcau O, Stefanescu H, Benea A, Hadade A, Margarit S, Sparchez Z, Tantau M, Ionescu D and Procopet B: Are presepsin and resistin better markers for bacterial infection in patients with decompensated liver cirrhosis? Dig Liver Dis 51: 1685-1691, 2019

32. Johnston CJ, Smyth DJ, Dresser DW and Maizels RM: TGF- $\beta$ in tolerance, development and regulation of immunity. Cell Immunol 299: 14-22, 2016.

33. Marcoe JP, Lim JR, Schaubert KL, Fodil-Cornu N, Matka M, McCubbrey AL, Farr AR, Vidal SM and Laouar Y: TGF- $\beta$ is responsible for NK cell immaturity during ontogeny and increased susceptibility to infection during mouse infancy. Nat Immunol 13: 843-850, 2012.

34. Meadows SK, Eriksson M, Barber A and Sentman CL: Human NK cell IFN-gamma production is regulated by endogenous TGF-beta. Int Immunopharmacol 6: 1020-1028, 2006.

35. Dong C: TH17 cells in development: An updated view of their molecular identity and genetic programming. Nat Rev Immunol 8: 337-348, 2008.

36. Lee YS, Park JS, Jung SM, Kim SD, Kim JH, Lee JY, Jung KC, Mamura M, Lee S, Kim SJ, et al: Inhibition of lethal inflammatory responses through the targeting of membrane-associated Toll-like receptor 4 signaling complexes with a Smad6-derived peptide. EMBO Mol Med 7: 577-592, 2015.

37. Park BS and Lee JO: Recognition of lipopolysaccharide pattern by TLR4 complexes. Exp Mol Med 45: e66, 2013.
38. Choi KC, Lee YS, Lim S, Choi HK, Lee CH, Lee EK, Hong S, Kim IH, Kim SJ and Park SH: Smad6 negatively regulates interleukin 1-receptor-Toll-like receptor signaling through direct interaction with the adaptor Pellino-1. Nat Immunol 7: 1057-1065, 2006

39. Ng PC, Chan KY, Leung KT, Tam YH, Ma TP, Lam HS, Cheung HM, Lee KH, To KF and Li K: Comparative MiRNA Expressional profiles and molecular networks in human small bowel tissues of necrotizing enterocolitis and spontaneous intestinal perforation. PLoS One 10: e0135737, 2015.

40. Staloch D, Gao X, Liu K, Xu M, Feng X, Aronson JF, Falzon M, Greeley GH, Rastellini C, Chao C, et al: Gremlin is a key profibrogenic factor in chronic pancreatitis. J Mol Med (Berl) 93: 1085-1093, 2015

41. Miao H, Wang N, Shi LX, Wang Z and Song WB: Overexpression of mircoRNA-137 inhibits cervical cancer cell invasion, migration and epithelial-mesenchymal transition by suppressing the TGF- $\beta$ /smad pathway via binding to GREM1. Cancer Cell Int 19: 147, 2019.

42. Mori T, Takahashi K, Naito M, Kodama T, Hakamata H, Sakai M, Miyazaki A, Horiuchi S and Ando M: Endocytic pathway of scavenger receptors via trans-Golgi system in bovine alveolar macrophages. Lab Invest 71: 409-416, 1994

43. Xu Z, Xu L, Li W, Jin X, Song X, Chen X, Zhu J, Zhou S, Li Y, Zhang $\mathrm{W}$, et al: Innate scavenger receptor-A regulates adaptive T helper cell responses to pathogen infection. Nat Commun 8: 16035, 2017.

44. Thomas CA, Li Y, Kodama T, Suzuki H, Silverstein SC and El Khoury J: Protection from lethal gram-positive infection by macrophage scavenger receptor-dependent phagocytosis. J Exp Med 191: 147-156, 2000.

45. Arredouani MS, Yang Z, Imrich A, Ning Y, Qin G and Kobzik L: The macrophage scavenger receptor SR-AI/II and lung defense against pneumococci and particles. Am J Respir Cell Mol Biol 35: 474-478, 2006

46. Wang J, Nikrad MP, Travanty EA, Zhou B, Phang T, Gao B, Alford T, Ito Y, Nahreini P, Hartshorn K, et al: Innate immune response of human alveolar macrophages during influenza $\mathrm{A}$ infection. PLoS One 7: e29879, 2012.

This work is licensed under a Creative Commons Attribution-NonCommercial-NoDerivatives 4.0 International (CC BY-NC-ND 4.0) License. 